Published in final edited form as:

Nat Cell Biol. 2013 February ; 15(2): 143-156. doi:10.1038/ncb2656.

\title{
Intracellular lumen extension requires ERM-1-dependent apical membrane expansion and AQP-8-mediated flux
}

\author{
Liakot A. Khan ${ }^{1}$, Hongjie Zhang ${ }^{1}$, Nessy Abraham ${ }^{1}$, Lei Sun ${ }^{3,4}$, John T. Fleming ${ }^{1}$, Matthew \\ Buechner $^{2}$, David H. Hall ${ }^{3}$, and Verena Gobel ${ }^{1}$ \\ ${ }^{1}$ Department of Pediatrics, Massachusetts General Hospital and Harvard Medical School, Boston, \\ Massachusetts 02114, USA \\ ${ }^{2}$ Department of Molecular Biosciences, University of Kansas, Lawrence, Kansas, 66045 USA \\ ${ }^{3}$ Center for C. elegans Anatomy, Department of Neuroscience, Albert Einstein College of \\ Medicine, Bronx, New York 10461, USA
}

\section{SUMMARY}

Many unicellular tubes such as capillaries form lumens intracellularly, a process that is not well understood. Here we show that the cortical membrane organizer ERM-1 is required to expand the intracellular apical/lumenal membrane and its actin undercoat during single-cell C.elegans excretory canal morphogenesis. We characterize AQP-8, identified in an ERM-1 overexpression (ERM-1[++]) suppressor screen, as a canalicular aquaporin that interacts with ERM-1 in lumen extension in a mercury-sensitive manner, implicating water-channel activity. AQP- 8 is transiently recruited to the lumen by ERM-1, co-localizing in peri-lumenal cuffs interspaced along expanding canals. An ERM-1[++]-mediated increase in the number of lumen-associated canaliculi is reversed by AQP- 8 depletion. We propose that the ERM-1-AQP-8 interaction propels lumen extension by translumenal flux, suggesting a direct morphogenetic effect of water-channel-regulated fluid pressure.

\section{INTRODUCTION}

Epithelial tubes are polarized and form a lumenal surface by their apical membranes. Multicellular tubes build the lumen at the interface of several cells, whereas unicellular tubes of the capillary type generate the lumen within the cell ${ }^{1,2}$. The C.elegans excretory cell extends four canals along the length of the animal from a single cell ${ }^{3,4}$. This unique in vivo model for intracellular lumen morphogenesis permits the separation of apical from

\footnotetext{
Users may view, print, copy, download and text and data- mine the content in such documents, for the purposes of academic research, subject always to the full Conditions of use: http://www.nature.com/authors/editorial_policies/license.html\#terms

Correspondence: gobel@helix.mgh.harvard.edu.

${ }^{4}$ Current address: Institute of Biophysics, Chinese Academy of Sciences, 15 Datun Road, Bejing, China 100101 AUTHOR CONTRIBUTIONS

L.A.K. performed experiments, generated, analyzed and assembled most of the data and contributed to project design and manuscript writing. H.Z. and N.A. contributed to the generation of transgenic strains and RNAi experiments. L.S. and D.H.H. performed the TEM and tomographic analyses, and J.T.F. contributed to TEM experiments. M.B. generated the canal-specific endosomal marker strains, and J.T.F. and M.B. contributed to writing of the manuscript. V.G. conceived and directed the project, participated in experiments and wrote the manuscript.
} 
basal membrane biogenesis and junction assembly (see below) $)^{5,6,7}$. It is not known how the canal lumen is built. A number of genes, mostly identified by their cyst-forming mutant canal phenotypes, are implicated in preserving stability of the lumenal membrane and its submembraneous cytoskeleton ${ }^{4,8-12}$.

We previously found that ERM-1, the single C.elegans orthologue of the ezrin-radixinmoesin (ERM) family of membrane-actin linkers, is enriched at lumenal membranes and required for tubular organ lumen morphogenesis, a function conserved in vertebrates ${ }^{13,14}$. ERMs are ubiquitous cortical membrane organizers with presumed structural as well as signaling functions, and they can initiate the formation of apical membrane characteristics such as microvilli in polarized cells ${ }^{15-17}$. It is still unclear, however, whether ERMs organize membranes via mechanical scaffolding or dynamic modeling, and whether they affect epithelial morphogenesis via junctions. ERMs' role in multicellular tubulogenesis, also conserved in invertebrates, has been difficult to dissect: Drosophila Dmoesin and zebrafish moesinl were implicated in both tube elongation and restriction, in both a junctiondependent and -independent manner ${ }^{18-20}$.

We took advantage of the simplicity of the single-cell C.elegans excretory canal to investigate ERMs' role in apical membrane modeling and lumen morphogenesis. We searched for genetic modifiers of the ERM-1 overexpression (ERM-1[++]) cystic-canal phenotype ${ }^{13}$ and identified aqp-8 (aquaporin), a member of the highly conserved family of membrane water channels. ${ }^{21}$ While aquaporin channel structure, permeability properties, and inhibition by mercury are well characterized in vitro, aquaporins' in vivo roles are less clear $^{22,23}$, since knockouts revealed non-essential roles for most. Given their tissue and organelle-specific localization, this finding is not explained by redundancies alone. Moreover, multiple knockouts have yielded few phenotypes: for instance, C.elegans $a q p-2 ; a q p-3 ; a q p-4 ; a q p-8$ quadruple null mutants are viable and superficially wild-type ${ }^{24}$.

Here we report a cooperative function of ERM-1 and AQP-8 in intracellular lumen and tube extension. Our findings suggest that ERM-1 extends intracellular lumens by: (1) expansion of the apical/lumenal membrane and its submembraneous cytoskeleton; (2) transient lumenal recruitment of AQP-8-associated canaliculi promoting translumenal flux.

\section{RESULTS}

\section{ERM-1 is required for intracellular lumenal membrane and actin coat expansion}

During late embryogenesis, the C.elegans excretory cell laterally extends two canal arms, each of which bifurcates and completes anterior-posterior extension in first-stage larvae (L1larvae). Canals subsequently expand synchronously with the animal's growth ${ }^{25}$ (Fig. 1a). The thin tubular canal arms ('canal-tubes', tube cytoplasm pseudocolored blue throughout) are surrounded by basal membranes and pierced by ultra-thin tubular lumens that are lined by lumenal membranes and a submembranous cytoskeleton of apical character ${ }^{10,13}$ ('canallumens; lumenal membranes shown in green to avoid confusion with 'canal-tubes' that also appear as single or double lines by fluorescence microcsopy; Fig. 1b, S1A-H). One junction connects the excretory cell to the duct, a connection essential for outflow and survival, but no junctions form along the length of the canal arms ${ }^{5,6}$. 
To assess ERM-1's role in canal morphogenesis, we examined its development under conditions of decreased and increased ERM-1. ERM-1::GFP appearance coincided with lumen initiation (1.5-fold embryo), was located at the lumenal membrane (Fig. S1I-J", 5ai”'), and led its expansion (Fig. 1c-h'). Cytoplasmic ERM-1::GFP puncta were never observed, suggesting that lumenal membranes do not expand from vacuole membranes with full apical character.

Canal extension, visualized with vha-1p- or sulp-5p-driven cytoplasmic excretory-canal GFP, was dependent on ERM-1 dosage: (1) progeny of erm-1(tm677) deletion mutants failed to extend canals and accumulated fluid in canal cell bodies of L1-arrested larvae (penetrance 100\%, N>200; Fig. 1i-i”); (2) homozygous progeny of tm677/+ animals also failed to extend canals and exhibited canal cell bodies that stretched laterally and posteriorward (penetrance 100\%, N>200; Fig. 1j-j"), suggestive of intact external guidance cues; (3) erm-1(tm677)/+ animals extended canals to $1 / 2$-wild-type length (penetrance $100 \%$; N>200; Fig. 1k-k"); (4) erm-1(RNAi) animals displayed a dose-dependent range from no to almost full-length canal extension (Fig. 1m-x). In all cases, the canal-to-duct junction remained intact, as indicated by proper localization of the junction component AJM-16,26 and by the ability of moderately ERM-1-depleted animals with short canals to survive to adulthood, suggesting that erm- 1 did not affect canal extension via junction assembly (Fig. S2A-C).

In ERM-1-depleted animals, the canal cytoplasm contained either vacuoles and no lumen, aligned discontiguous vacuoles, or vacuolar-shaped shortened lumens (Fig. 1m-r, S2D-I). Where vacuoles were misaligned, at canal tips (Fig. 1p, S2G) or in the cell body (Fig. 1n, $\mathrm{S} 2 \mathrm{E}$ ), lumens did not extend or deviated from their course, suggestive of a link between vacuole alignment and directional extension. To examine lumenal membrane expansion, canals were labeled with ACT-5::GFP, a tube-specific actin that, like ERM-1, was found to reside at lumenal membranes ${ }^{13,27}$. Vacuole alignment correlated with lumenal actin assembly (Fig. 1s-x, S2J-O): isolated vacuoles lacked ACT-5::GFP (Fig. 1s, S2J); aligned vacuoles were partially coated (Fig. 1t, S2K); and vacuolar-shaped lumenal membranes were fully coated with ACT-5::GFP (Fig. 1v,w, S2M,N).

ERM-1 overexpression (ERM-1[++]) from a transgenic erm-1 allele (fgIs2[erm-1p::erm-1]) ${ }^{13}$ and several fgEx(erm-1p:::erm-1::gfp) lines resulted in widened and deformed canal lumens (100\%, N>1000; Fig. 2a-f, S3A-F). fgIs2(erm-1p::erm-1), previously shown to fully rescue $t m 677^{13}$, raised erm- 1 mRNA $\sim 8$ fold and ERM-1 protein (detected by distinct monoclonal and polyclonal antibodies) 9fold, and was suppressed by erm-l RNAi (100\%, N>500; Fig. 2g-h; S3G-J, not shown), all data indicating that higher amounts of functional ERM-1 caused the defect. erm-1(RNAi) only suppressed ERM-1[++]induced cysts when initiated before or during active lumen extension (Fig. 2o).

Nomarski and confocal analysis of GFP-labeled ERM-1[++] canals revealed cystic fluid buildup in short canals that extended lumenless cytoplasmic islets beyond tip cysts (Fig. 2b,c). An ACT-5::GFP coat characterized the fluid buildup as intralumenal cysts (intralumenal spheres, bounded by apical membranes, are denoted as cysts; small and large cytoplasmic spheres, not fully bounded by such membranes, are denoted as 'vesicles' and 
'vacuoles', respectively). Unlike wild-type ACT-5 coats, ERM-1[++] ACT-5 coats extended beyond the lumen and were thicker (Fig. 2d-f, S3K-O). Thus, ERM-1[++] canals display excess/aberrant lumenal actin coating and form intralumenal cysts, whereas ERM-1depleted canals display no or incomplete lumenal membrane expansion with discontiguous actin coating and accumulate cytoplasmic vacuoles.

We conclude that ERM-1 dose-dependently expands the junctionless C.elegans excretory canal lumenal membrane and its actin cytoskeleton. ERM-1 depletion phenotypes are compatible with a vacuole-coalescence model of canal lumen extension and suggest that lumen-forming vesicle membranes concomitantly align, coalesce, and acquire apical characteristics and a submembraneous actin track that directionally extends an intracellular lumen.

\section{An RNAi modifier screen reveals a requirement of AQP-8/aquaporin for the ERM-1[++] excretory canal phenotype}

To determine the molecular basis of ERM-1's role in intracellular lumen extension, we performed a targeted RNAi ERM-1[++] modifier screen. An RNAi library was built from experimentally identified ERM/ezrin-radixin-moesin interactors from all species, and eight classes of candidates were generated and extended by mining available databases (Fig. 2i). Corresponding C.elegans orthologues $(\mathrm{N}=1300)$ were knocked down via feeding RNAi bacteria to an ERM-1[++] strain with vha-1p::GFP-labeled canals. Enhancement was defined as increased cyst size or canal shortening, and suppression as reduced cyst size and canal re-extension (Fig. 2j-m). aqp-8 was one of several genes identified as a strong suppressor of the ERM-1[++] cystic-canal phenotype (Tab. S1).

$a q p-8(R N A i)$ suppression was confirmed in $a q p-8($ ok2800), a likely null allele deleting part of the gene and introducing a frameshift and stop codon (Fig. S5A). Over $40 \%$ of aqp-8(ok2800); fgIs(erm-1p::erm-1) double mutants had thinner and re-extended canals, without cysts, as compared to animals containing only fgIs2(erm-1p::erm-1) (Fig. 2n, S4AB'). This aqp-8 mutation, or aqp-8 RNAi, also suppressed overexpression phenotypes of erm-1::gfp transgenic strains (not shown). Suppression was specific to ERM-1[++] cysts, as exc-5(rh232), exc-4(rh133), and exc-2(rh90) cysts ${ }^{4,10}$ were not suppressed (Fig. S4C-E). Feeding animals aqp-8 double-stranded RNA after completion of embryogenesis, sufficient to abolish AQP-8::GFP, suppressed ERM-1[++] cysts only when introduced at the L1-L2stage (Fig. 2o, Fig S4F-G').

We conclude that AQP-8 interacts with ERM-1 in canal development and exerts its effect during the phase of active lumen and canal extension.

\section{AQP-8 contributes to lumen and canal extension and is expressed on canalicular vesicles}

To investigate AQP-8's role in excretory canal morphogenesis we examined canal development after modulating AQP-8 levels and analyzed AQP-8's subcellular localization. Viable mutants in two different likely null alleles, aqp-8(ok2800) and aqp-8(tm1919), labeled with either cytoplasmic- or lumenal-GFP, exhibited moderately shortened canals and lumen defects at $\sim 40 \%$ penetrance, including: canal-tip vacuoles; extension of cytoplasmic 
islets beyond prematurely-ending lumens; and reduced lumen diameters (Fig. 3a-e, S1D-H, S5B, not shown). aqp-8 knockdowns generated a range of mild to moderate canal defects of similar character, with strong RNAi phenocopying the germline deletion mutants. Mutant canal phenotypes were rescued by transformation with either $a q p-8 p-a q p-8:: g f p$ or $a q p-8 p$ aqp-8::mCherry plasmids (Fig. 3f, S5B).

AQP-8 overexpression (AQP-8[++]) from several independent lines caused widened canals (penetrance $\sim 90 \%, \mathrm{~N}>500$ ) or round intralumenal cell-body-close cysts, without canal extension, and lethality (penetrance $\sim 10 \%, \mathrm{~N}>500$; Fig. 3g-h, S5C-E'). Increased copies of $a q p-8 p:: a q p-8:: g f p$ (the $a q p-8(o k 2800)$ rescuing plasmid) raised $a q p-8$ mRNA levels and progressively widened canals, and AQP-8[++] defects were suppressed by aqp- 8 RNAi (Fig. $3 \mathrm{i}$, S5F, not shown). These data indicate that, similar to ERM-1, higher levels of functional AQP-8 also affect canal morphogenesis.

To examine possible redundancies of AQP-8 with other water channels in canal development, we inspected GFP-labeled canals in animals depleted of other C.elegans aquaporins (Fig. S6). aqp-2, aqp-3 and aqp-8 are the three C.elegans aquaporins shown to be expressed in the excretory canal ${ }^{24,28}$. An aqp-2(ok2159) presumed null allele failed to generate obvious canal defects, as did the aqp-2(ok2159);aqp-3(RNAi) double deletion/ knockdown that also failed to enhance the $a q p-8(R N A i)$ phenotype (Fig. S6A). None of the other tested aquaporin knockdowns generated discernible defects, and among them only $a q p-8$ suppressed the ERM-1[++] phenotype (Fig. S6B-C).

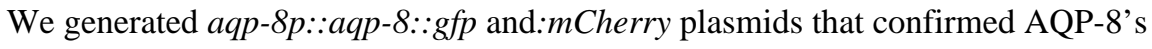
expression in the excretory system ${ }^{24,28}$ and revealed it as an endo-rather than plasmamembrane-associated channel that localized to dense cytoplasmic, mostly lumen-distant puncta/patches (Fig. 3j-v', 5f,i-i'”, S5G,H; Fig. S7 for additional AQP-8 localization). This expression pattern suggested that AQP-8 resides on canaliculi that fill the canal cytoplasm as small vesicles whose inter-connection and lumen-connection may be dynamically regulated (Fig. 3w-x,7h) ${ }^{5}$. AQP-8::GFP did not overlap with mCherry::RAB-11,:RAB-5,:RME-1,:CHC-1, identifying larger, sparser, and basally aligned endosomal vesicles ${ }^{29}$ (Fig. 3m-p', 7h-k). We generated GFP-fusions with VHA-1, a vacuolar ATPase subunit likely located on canaliculi, that copied the AQP-8 expression pattern and partially colocalized (Fig. 3q-s'). AQP-8 was also partially collocated with GFP and mCherry fusions to VHA-5, another component of the vacuolar ATPase, shown via immuno-electronmicroscopy to be located at canaliculi ${ }^{7}$ (Fig. 3t-v').

We conclude that AQP-8 is a canalicular-vesicle-rather than plasma-membrane-associated aquaporin in the C.elegans excretory canal and likely the sole aquaporin to function in lumen and canal extension. AQP-8, like ERM-1, dose-dependently extends canal lumens, but, unlike ERM-1, seems to expand lumen diameters rather than membranes.

\section{Genetic interactions between AQP-8 and ERM-1}

To further investigate the nature of the AQP-8/ERM-1 interaction, we examined canal extension and cyst formation in different combinations of erm- 1 and aqp- 8 loss- and gainof-function conditions. aqp- $8(\mathrm{ok} 2800)$ enhanced the partial loss-of-function erm-l(RNAi) 
canal defects, supporting a coordinate function of these genes in canal extension (Fig. 4a). Since homozygous erm-1 (tm677) mutants extend no canals, further reduction of length cannot be measured. However, erm-1(tm677);aqp-8(ok2800) double-mutant canal-cell bodies contained fewer vacuoles, suggesting an ERM-1-independent function of AQP-8 in intracellular vesicle swelling (Fig. 4b).

ERM-1[++] enhanced the AQP-8[++] cystic-canal phenotype, also supporting their common function in canal lumen morphogenesis (Fig. 4c). Unexpectedly, however, erm-1 RNAi suppressed AQP-8[++]-induced cyst formation, indicating that it requires ERM-1 (Fig. 4d). Loss of ERM-1, a lumenal scaffold component, would be predicted to enhance canal cysts, as indeed observed for RNAi with $s m a-1 / \beta_{\mathrm{H}^{-}}$-spectrin, encoding another canal-lumenscaffold constituent ${ }^{4}$, that enhanced AQP-8[++] cysts (Fig. 4e).

We conclude that $e r m-1$ and $a q p-8$ genetically interact in excretory canal lumen extension, and that they are mutually required to generate their overexpression cystic canal phenotypes, a scenario compatible with a direct, possibly physical, ERM-1/AQP-8 interaction in this process. aqp- 8 appears to have an additional $\mathrm{erm}$ - 1 -independent function in excretory-canalvesicle swelling.

\section{AQP-8 and ERM-1 colocalize in peri-lumenal cuffs during canal lumen extension and interact in yeast-two-hybrid assays}

Given AQP-8 and ERM-1's distinct location at endo-versus plasma membranes, a direct in vivo AQP-8/ERM-1 interaction seemed unlikely. To explore the possibility of a spatially or temporally restricted interaction, we examined co-expressed AQP-8::mCherry and ERM-1::GFP throughout canal development. AQP-8::mCherry appeared only after completion of lateral canal extension, lagging behind lumenal ERM-1::GFP, indicating that AQP-8, unlike ERM-1, is not strictly required for lumen extension (Fig. 5a-b'). AQP-8::mCherry then rapidly spread along canal arms, overtaking ERM-1 at canal tips by the time of anterior-posterior canal extension (Fig. 5c-d'). AQP-8 expression dramatically increased in canal varicosities, excess canal tissue that forms along actively growing canals $^{30}$ and during an osmotic challenge ${ }^{7}$ (Fig. 5e). AQP-8::mCherry/ERM-1::GFP lumenal overlap was restricted to this growth phase and condensed into peri-lumenal cuffs through enrichment in varicosities (Fig. 5g-h"'). Both AQP-8 expression and ERM-1 overlap sharply declined with the flattening of varicosities, when AQP-8 assumed its adult lumen-distant vesicular expression pattern (Fig. 5f,i-i'”). Varicosities and AQP-8::mCherry/ ERM-1::GFP overlap persisted at adult posterior canal tips, suggesting their role in lumen tip maintenance (Fig. S8A-C').

To examine a possible physical interaction of ERM- 1 and AQP- 8 we performed yeast twohybrid assays. ERM-1 interacted with AQP-8, as well as with ACT-5/actin, but not with UNC-54/myosin (Fig. 5j-m, S8D-D'). Mapping of ERM-1 domains suggested that AQP-8 binds to the C-terminal ERM-1 domain.

We conclude that a putative physical ERM-1/AQP-8 interaction is temporally and spatially regulated in vivo and largely confined to the short period of active canal extension and to peri-lumenal cuffs at canal varicosities interspaced along extending canals. 


\section{Mercury inhibits AQP-8's effects on excretory canal morphogenesis and the ERM-1[++] phenotype}

As a water channel, AQP-8 might extend the canal lumen by translumenal flux. However, not all aquaporins transport water ${ }^{31}$ and AQP-8 fails to do so under isotonic conditions in Xenopus oocytes ${ }^{24}$. We reasoned that it might nevertheless transport water into acidified vesicles or intracellular lumens. To test this, we first assayed osmotic behavior in aqp-8 mutants. To target the efferent arm of osmoregulation, worms were directly placed into nonisotonic solutions, rather than allowing adjustment to different plate osmolalities ${ }^{24}$. Under these conditions, aqp-8(ok2800) mutants were sensitive to hypotonic stress (Fig. 6a-c).

Next, we examined the effect of mercury that specifically inhibits aquaporin water-channel activity ${ }^{32}$. Mercury at non-toxic doses mildly inhibited excretory canal extension in otherwise wild-type-appearing animals (Fig. 6d). This effect was largely mediated by AQP-8, since mercury failed to enhance $a q p-8(o k 2800)$-induced excretory-canal shortening (Fig. 6e). Moreover, non-toxic mercury doses substantially suppressed AQP-8[++]-induced cyst formation and partially suppressed AQP-8[++]-induced canal shortening (Fig. 6f,g,h,k). These data indicate that AQP- 8 acts as a water channel in canal morphogenesis, and suggest that excess flux during canal extension expands lumen diameter at the expense of forward extension.

ERM-1[++]-induced cysts and canal shortening were likewise suppressed by mercury, albeit only partially (Fig. 6i-k), indicating that water flux contributes to ERM-1[++] cysts, that ERM-1 affects such flux, and that AQP-8 depletion might suppress ERM-1[++] cysts via the reduction of flux. In support of this idea, mercury reduced AQP-8[++]'s ability to enhance ERM-1[++] cysts ( $16 \%$ cyst reduction, $\mathrm{N}=70$ ). Mercury specifically affected AQP-8[++] and ERM-1[++] cysts, as exc-5(rh232) cysts could not be suppressed (Fig. 6k). Cysts were suppressed only when mercury was present during active canal extension (Fig. 61).

To further determine AQP-8's morphogenetic function as a water channel, we mutated conserved residues in the channel region required for water transport, including a mutation that induces diabetes insipidus in humans ${ }^{33}$, and examined their effects on canal extension.

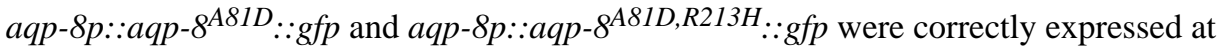

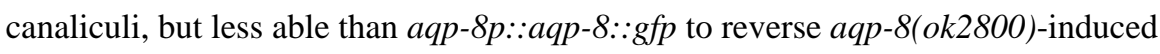
excretory-canal defects and the aqp-8(ok2800)-induced suppression of ERM-1[++] cysts (Fig. 6m-o).

We conclude that water flux has a direct morphogenetic effect on unicellular tubes and that AQP-8 extends intracellular lumens by its ability to transport water, which also mediates its interaction with ERM-1.

\section{ERM-1 recruits AQP-8 to the lumen and increases the canaliculi-lumen connection}

AQP-8-mediated flux could directly extend lumens by influx either into the lumen or into subsequently lumen-connecting vesicles. Alternatively, vesicular influx might extend lumens secondarily, through effects on the vesicular delivery of lumenal membrane components. To explore these possibilities in relation to an ERM-1/AQP-8 interaction, we examined whether AQP-8 was required for lumenal recruitment of ERM-1 or vice versa. 
aqp-8(ok2800) mutants recruited both ERM-1::GFP and ACT-5::GFP to the expanding lumenal membrane (Fig. 7a,b). In contrast, AQP-8::GFP, AQP-8::mCherry and VHA-1::GFP tubulovesicles remained dispersed away from extending lumens of erm-1(tm677)/+ and erm-1(RNAi) animals, but not from sma-1(RNAi) lumens, which also exhibit lumen morphogenesis defects (Fig. 7c-e, S8E,F).

In addition, surplus ERM-1 recruited AQP-8::mCherry puncta to the lumen of mature canals, where they accumulated in bright patches, and, in contrast to wild-type, overlapped with lumenal ACT-5::GFP (Fig. 7f-g”, Fig. S8I, Tab. S2). The AQP-8/ACT-5 accretion was not caused by physical constraint of the cysts, as indicated by the unaltered homogenous distribution of cytoplasmic sulp-5p::GFP (Fig. S8G-I).

Comparisons of high-pressure-freezing-transmission-electron-microsocopic cross sections of ERM-1[++] versus wild-type canals revealed cystic lumens with undulating membranes framing cytoplasmic bulges. In these bulges, the tubovesicluar membrane system was shifted towards the lumen, with an increase in the fraction of lumen-connected vesicles (from 10-40\%), in vesicle interconnections and vesicle density, and with the appearance of dramatically elongated lumen-connected tubules (Fig. 7h,i; 8a,c; S8J, Tab. S3).

Tomographic analysis confirmed the increase in lumen-connected canaliculi in ERM-1[++] canals (Fig. 8a-d). This increase was alleviated in aqp-8(ok2800); ERM-1[++] canals (Fig. $7 \mathrm{k}, \mathrm{S} 8 \mathrm{~J}, \mathrm{Tab} . \mathrm{S} 3)$. In contrast, aqp-8(ok2800) canal cross sections displayed a decreased fraction of interconnected canalicular vesicles (from 14 to $3 \%$ ) and a moderate reduction in lumen diameter (Fig. 7j, S8K, Tab. S3).

We conclude that ERM-1 recruits AQP-8 to the excretory canal lumen and excess ERM-1 increases the canalicular-lumenal interface in an AQP-8-dependent manner.

\section{DISCUSSION}

Two models for intracellular lumen extension by lumenal membrane expansion have been proposed. Zebrafish intracellular and intercellular vascular lumens extend by coalescing vacuolar membranes of presumed apical character, elaborating the in vitro capillarogenesis model $^{20,34-36}$. It is unclear how this process acquires intracellular directionality. Moesin1 promotes lumen extension, but is thought to exert its effect secondarily via intercellular junctions and junction-dependent polarization ${ }^{20}$. In contrast, in Drosophila terminaltracheal-branch morphogenesis, an intracellular lumen extends through inward growth of the apical membrane along a pre-established actin track, without apparent vacuolar fusions ${ }^{19}$. The actin track contains Dmoesin, but its function is unclear.

The single-cell C.elegans excretory canal permits the observation of a direct ERM-1 role in the expansion of an intracellular lumenal membrane and its actin undercoat, and furthermore suggests that it occurs through coalescence of vesicle membranes that acquire apical character and a directional actin track during, not before, coalescence. This could propose a unifying model for intracellular lumenal membrane expansion: the coalescence of different vesicle membranes, not necessarily of apical character (and thus not detected by apical labels in Drosophila and C.elegans), with concomitant actin track construction (perhaps also 
present in zebrafish). Indeed, vesicle trafficking is also required for Drosophila terminaltracheal-branch morphogenesis ${ }^{37}$.

ERM-1's ability to expand a junctionless apical/lumenal membrane now demonstrates a direct ERM requirement for de novo apical membrane and lumen biogenesis and reveals its junction-independent function in this process. ERM-1's function instead consists of the recruitment and actin-coating of membrane-forming vesicles, raising the possibility that ERMs regulate cortical membrane dynamics by negotiating vesicle-to-plasma-membrane contacts (perhaps via actin) rather than by membrane-scaffolding ${ }^{15}$, consistent with recent reports on ERMs' role in vesicle dynamics ${ }^{38-41}$.

In support of an ERM role at the vesicle-to-membrane interface, we also find that ERM-1 recruits AQP-8-associated canalicular vesicles to the lumenal membrane. However, lumenal membranes do not incorporate AQP-8, suggesting that these vesicles only transiently connect, a scenario compatible with an AQP-8 function in lumen extension via flux. Nonetheless, even a transient canaliculi-membrane connection modifies the lumenal cortex. This may explain dual ERM functions on membrane channels/pumps previously observed in other systems, such as effects on both the pump function of membrane-associated $\mathrm{Na} / \mathrm{H}$ exchangers (NHE3) and on the structural, pump-independent function on cortex organization by NHE1, as well as its effect on increasing gastric acid secretion via both $\mathrm{H}$ +/K+-ATPase-activation and the expansion of a microvillar-canalicular interface ${ }^{42-46}$.

Most ERM interactors associate with ERMs' membrane-directed N-terminus ${ }^{15}$. An AQP-8 interaction with ERM-1's actin-binding C-terminus might generate a canaliculi-membrane bridge akin to its actin-membrane link. Intriguingly, a C-terminal Moesin peptide promotes apical membrane recruitment of AQP2 in mammalian cell lines ${ }^{47}$. This was interpreted as an inhibitory peptide effect, but perhaps reflects a peptide-dependent recruitment of aquaporin to the membrane. It is tempting to speculate that AQP-8 competes with actin for ERM-1's $\mathrm{C}$-terminus during canal extension, resulting in transient canaliculi-membrane contacts at the expense of actin-membrane coating. ERM-1 might thus orchestrate lumenal membrane expansion with lumen diameter expansion (Fig. 8e,f).

A direct, regulated and active morphogenetic force of hydrostatic pressure in metazoan development has long been proposed, but not yet demonstrated. ${ }^{48}$ However, hydrostatic pressure was recently shown to shape cells in vitro ${ }^{49}$, and to indirectly shape developing zebrafish tissues, such as nephrons, via cell migration, and vessels via lumen stabilization and anastomosis. ${ }^{20,36,50}$ It can also passively expand preformed developing structures, for instance, the zebrafish gut via CFTR-channel-mediated flux or, as paracellular flux, single lumens in Xenopus multicellular tubes ${ }^{51-53}$. In contrast, hydrostatic pressure is a documented direct, active and, moreover, aquaporin-regulated tissue-shaping force in plants (that harbor a greater variety of vesicular aquaporins) ${ }^{54}$. No such role has yet been shown for metazoan aquaporins, although their function in development is accumulating: AQP11deficient mice develop polycystic kidneys for unknown reasons ${ }^{55}$; mouse AQP1 and AQP2 support tumor angiogenesis and renal tubulogenesis, respectively, via cell migration ${ }^{56,57}$; and aquaporins affect morphogenesis flux-independently, via adhesion, for instance lens 
AQP0 (functioning as a junction in its closed configuration) and the Drosophila AQP4 homolog big brain ${ }^{31,58-60}$.

The present analysis now suggests that water-channel-regulated translumenal flux can also act as a direct tissue-shaping force in metazoan development, specifically in intracellular lumen extension. Aquaporins are known to shuttle between vesicular and plasma membranes, or move onto secretory vesicles, with or without incorporation into the plasma membrane ${ }^{61-64}$. If the AQP-8-mediated translumenal flux results from water flow into subsequently lumen-connecting canalicular vesicles, vesicular ions may contribute to its morphogenetic effect, consistent with the prior implication of ion channels in tubulogenesis and supported by the accompanying paper ${ }^{7,10,65}$. The tight temporal and spatial regulation of the ERM-1-AQP-8 interaction fits with a morphogenetic purpose of this flux. The provocative restriction of this interaction to peri-lumenal cuffs, spaced in periodic intervals along expanding canals, could suggest that localized fluid pulses aid in propelling active lumen extension (Fig. 8g).

\section{Supplementary Material}

Refer to Web version on PubMed Central for supplementary material.

\section{ACKNOWLEDGEMENTS}

We thank D. Baillie (Simon Fraser University, Burnaby, British Columbia, Canada), M. Futai (Osaka University, Osaka, Japan), M. Labouesse (IGBMC, France), K. Nehrke (University of Rochester Medical Center, Rochester New York, USA), and J. Simske (Case Western Reserve University School of Medicine, Cleveland, Ohio, USA), and the following C. elegans resource centers: J. Kohara (National Institute of Genetics, Mishima, Japan), S. Mitani (National Bioresource Project, Tokyo Women's Medical University, Tokyo, Japan), the International C. elegans Gene Knockout Consortium and the Caenorhabditis elegans genetic center (NIH Center for Research Resources) for providing plasmids and strains. We thank E. Membreno and D. Fernandez for technical support, A. Sengupta for 3D arts, A. Kim for image editing, F. Solomon for critical reading of the manuscript, and H. Weinstein and A. Walker for ongoing support. This work was supported by NIH grant GM078653 and a Mattina R. Proctor Award to V.G.

\section{REFERENCES}

1. Lubarsky B, Krasnow MA. Tube morphogenesis: making and shaping biological tubes. Cell. 2003; 112:19-28. [PubMed: 12526790]

2. Bryant DM, Mostov KE. From cells to organs: building polarized tissue. Nat Rev Mol Cell Biol. 2008; 9:887-901. [PubMed: 18946477]

3. Nelson FK, Albert PS, Riddle DL. Fine structure of the Caenorhabditis elegans secretory-excretory system. J Ultrastruct Res. 1983; 82:156-171. [PubMed: 6827646]

4. Buechner M, Hall DH, Bhatt H, Hedgecock EM. Cystic canal mutants in Caenorhabditis elegans are defective in the apical membrane domain of the renal (excretory) cell. Dev Biol. 1999; 214:227241. [PubMed: 10491271]

5. Hall, DH.; Altun, ZF. C. elegans Atlas. Cold Spring Harbor Laboratory Press; New York: 2008.

6. Abdus-Saboor I, et al. Notch and Ras promote sequential steps of excretory tube development in C. elegans. Development. 2011; 138:3545-3555. [PubMed: 21771815]

7. Kolotuev, I.; Hyenne, V.; Schwab, Y.; Rodriguez, D.; Labouesse, M. A pathway for unicellular tube extension depending on the lymphatic vessel determinant Prox1 and on osmoregulation. http:// dx.doi.org/10.1038/ncb2662(2013)

8. Jones SJ, Baillie DL. Characterization of the let-653 gene in Caenorhabditis elegans. Mol Gen Genet. 1995; 248:719-726. [PubMed: 7476875] 
9. Suzuki N, et al. A putative GDP-GTP exchange factor is required for development of the excretory cell in Caenorhabditis elegans. EMBO Rep. 2001; 2:530-535. [PubMed: 11415987]

10. Berry KL, Bulow HE, Hall DH, Hobert OA. C. elegans CLIC-like protein required for intracellular tube formation and maintenance. Science. 2003; 302:2134-2137. [PubMed: 14684823]

11. Fujita M, et al. The role of the ELAV homologue EXC-7 in the development of the Caenorhabditis elegans excretory canals. Dev Biol. 2003; 256:290-301. [PubMed: 12679103]

12. Perens EA, Shaham S. C. elegans daf-6 encodes a patched-related protein required for lumen formation. Dev Cell. 2005; 8:893-906. [PubMed: 15935778]

13. Gobel V, Barrett PL, Hall DH, Fleming JT. Lumen morphogenesis in C. elegans requires the membrane-cytoskeleton linker erm-1. Dev Cell. 2004; 6:865-873. [PubMed: 15177034]

14. Saotome I, Curto M, McClatchey AI. Ezrin is essential for epithelial organization and villus morphogenesis in the developing intestine. Dev Cell. 2004; 6:855-864. [PubMed: 15177033]

15. Fehon RG, McClatchey AI, Bretscher A. Organizing the cell cortex: the role of ERM proteins. Nat Rev Mol Cell Biol. 2010; 11:276-287. [PubMed: 20308985]

16. ten Klooster JP, et al. Mst4 and Ezrin induce brush borders downstream of the Lkb1/Strad/Mo25 polarization complex. Dev Cell. 2009; 16:551-562. [PubMed: 19386264]

17. Zhu L, Crothers J Jr. Zhou R, Forte JG. A possible mechanism for ezrin to establish epithelial cell polarity. Am J Physiol Cell Physiol. 2010; 299:C431-443. [PubMed: 20505040]

18. Kerman BE, Cheshire AM, Myat MM, Andrew DJ. Ribbon modulates apical membrane during tube elongation through Crumbs and Moesin. Dev Biol. 2008; 320:278-288. [PubMed: 18585700]

19. Gervais L, Casanova J. In vivo coupling of cell elongation and lumen formation in a single cell. Curr Biol. 2010; 20:359-366. [PubMed: 20137948]

20. Wang Y, et al. Moesin1 and Ve-cadherin are required in endothelial cells during in vivo tubulogenesis. Development. 2010; 137:3119-3128. [PubMed: 20736288]

21. King LS, Kozono D, Agre P. From structure to disease: the evolving tale of aquaporin biology. Nat Rev Mol Cell Biol. 2004; 5:687-698. [PubMed: 15340377]

22. Preston GM, Jung JS, Guggino WB, Agre P. The mercury-sensitive residue at cysteine 189 in the CHIP28 water channel. J Biol Chem. 1993; 268:17-20. [PubMed: 7677994]

23. Hachez C, Chaumont F. Aquaporins: a family of highly regulated multifunctional channels. Adv Exp Med Biol. 2010; 679:1-17. [PubMed: 20666220]

24. Huang CG, Lamitina T, Agre P, Strange K. Functional analysis of the aquaporin gene family in Caenorhabditis elegans. Am J Physiol Cell Physiol. 2007; 292:C1867-1873. [PubMed: 17229810]

25. Buechner M. Tubes and the single C. elegans excretory cell. Trends Cell Biol. 2002; 12:479-484. [PubMed: 12441252]

26. Koppen M, et al. Cooperative regulation of AJM-1 controls junctional integrity in Caenorhabditis elegans epithelia. Nat Cell Biol. 2001; 3:983-991. [PubMed: 11715019]

27. MacQueen AJ, et al. ACT-5 is an essential Caenorhabditis elegans actin required for intestinal microvilli formation. Mol Biol Cell. 2005; 16:3247-3259. [PubMed: 15872090]

28. Mah AK, et al. Transcriptional regulation of AQP-8, a Caenorhabditis elegans aquaporin exclusively expressed in the excretory system, by the POU homeobox transcription factor CEH-6. J Biol Chem. 2007; 282:28074-28086. [PubMed: 17660295]

29. Mattingly BC, Buechner M. The FGD homologue EXC-5 regulates apical trafficking in C. elegans tubules. Dev Biol. 2011; 359:59-72. [PubMed: 21889936]

30. Hahn-Windgassen A, Van Gilst MR. The Caenorhabditis elegans HNF4alpha Homolog, NHR-31, mediates excretory tube growth and function through coordinate regulation of the vacuolar ATPase. PLoS Genet. 2009; 5:e1000553. [PubMed: 19668342]

31. Liu J, Xu J, Gu S, Nicholson BJ, Jiang JX. Aquaporin 0 enhances gap junction coupling via its cell adhesion function and interaction with connexin 50. J Cell Sci. 2011; 124:198-206. [PubMed: 21172802]

32. Agre P, Bonhivers M, Borgnia MJ. The aquaporins, blueprints for cellular plumbing systems. J Biol Chem. 1998; 273:14659-14662. [PubMed: 9614059]

33. Cheong HI, et al. Two novel mutations in the aquaporin 2 gene in a girl with congenital nephrogenic diabetes insipidus. J Korean Med Sci. 2005; 20:1076-1078. [PubMed: 16361827] 
34. Folkman J, Haudenschild C. Angiogenesis in vitro. Nature. 1980; 288:551-556. [PubMed: 6160403]

35. Kamei M, et al. Endothelial tubes assemble from intracellular vacuoles in vivo. Nature. 2006; 442:453-456. [PubMed: 16799567]

36. Herwig L, et al. Distinct cellular mechanisms of blood vessel fusion in the zebrafish embryo. Curr Biol. 2011; 21:1942-1948. [PubMed: 22079115]

37. Schottenfeld-Roames J, Ghabrial AS. Whacked and Rab35 polarize dynein-motor-complexdependent seamless tube growth. Nat Cell Biol. 2012; 14:386-393. [PubMed: 22407366]

38. Deretic D, et al. Phosphoinositides, ezrin/moesin, and rac1 regulate fusion of rhodopsin transport carriers in retinal photoreceptors. Mol Biol Cell. 2004; 15:359-370. [PubMed: 13679519]

39. Balklava Z, Pant S, Fares H, Grant BD. Genome-wide analysis identifies a general requirement for polarity proteins in endocytic traffic. Nat Cell Biol. 2007; 9:1066-1073. [PubMed: 17704769]

40. Chirivino D, et al. The ERM proteins interact with the HOPS complex to regulate the maturation of endosomes. Mol Biol Cell. 2011; 22:375-385. [PubMed: 21148287]

41. Kaksonen M, Toret CP, Drubin DG. A modular design for the clathrin- and actin-mediated endocytosis machinery. Cell. 2005; 123:305-320. [PubMed: 16239147]

42. Lamprecht G, Weinman EJ, Yun CH. The role of NHERF and E3KARP in the cAMP-mediated inhibition of NHE3. J Biol Chem. 1998; 273:29972-29978. [PubMed: 9792717]

43. Denker SP, Huang DC, Orlowski J, Furthmayr H, Barber DL. Direct binding of the Na--H exchanger NHE1 to ERM proteins regulates the cortical cytoskeleton and cell shape independently of $\mathrm{H}(+)$ translocation. Mol Cell. 2000; 6:1425-1436. [PubMed: 11163215]

44. Zhou R, et al. Characterization of protein kinase A-mediated phosphorylation of ezrin in gastric parietal cell activation. J Biol Chem. 2003; 278:35651-35659. [PubMed: 12840026]

45. Tamura A, et al. Achlorhydria by ezrin knockdown: defects in the formation/expansion of apical canaliculi in gastric parietal cells. J Cell Biol. 2005; 169:21-28. [PubMed: 15809309]

46. Cha B, et al. The NHE3 juxtamembrane cytoplasmic domain directly binds ezrin: dual role in NHE3 trafficking and mobility in the brush border. Mol Biol Cell. 2006; 17:2661-2673. [PubMed: 16540524]

47. Tamma $\mathrm{G}$, et al. Actin remodeling requires ERM function to facilitate AQP2 apical targeting. $\mathrm{J}$ Cell Sci. 2005; 118:3623-3630. [PubMed: 16046477]

48. Desmond ME, Jacobson AG. Embryonic brain enlargement requires cerebrospinal fluid pressure. Dev Biol. 1977; 57:188-198. [PubMed: 863106]

49. Stewart MP, et al. Hydrostatic pressure and the actomyosin cortex drive mitotic cell rounding. Nature. 2011; 469:226-230. [PubMed: 21196934]

50. Vasilyev A, et al. Collective cell migration drives morphogenesis of the kidney nephron. PLoS Biol. 2009; 7:e9. [PubMed: 19127979]

51. Bagnat M, Cheung ID, Mostov KE, Stainier DY. Genetic control of single lumen formation in the zebrafish gut. Nat Cell Biol. 2007; 9:954-960. [PubMed: 17632505]

52. Krupinski T, Beitel GJ. Unexpected roles of the Na-K-ATPase and other ion transporters in cell junctions and tubulogenesis. Physiology (Bethesda). 2009; 24:192-201. [PubMed: 19509129]

53. Bagnat M, et al. Cse11 is a negative regulator of CFTR-dependent fluid secretion. Curr Biol. 2010; 20:1840-1845. [PubMed: 20933420]

54. Maurel C, Verdoucq L, Luu DT, Santoni V. Plant aquaporins: membrane channels with multiple integrated functions. Annu Rev Plant Biol. 2008; 59:595-624. [PubMed: 18444909]

55. Morishita Y, et al. Disruption of aquaporin-11 produces polycystic kidneys following vacuolization of the proximal tubule. Mol Cell Biol. 2005; 25:7770-7779. [PubMed: 16107722]

56. Saadoun S, Papadopoulos MC, Hara-Chikuma M, Verkman AS. Impairment of angiogenesis and cell migration by targeted aquaporin-1 gene disruption. Nature. 2005; 434:786-792. [PubMed: 15815633]

57. Chen Y, et al. Aquaporin 2 Promotes Cell Migration and Epithelial Morphogenesis. J Am Soc Nephrol. 2012

58. Tatsumi K, et al. Drosophila big brain does not act as a water channel, but mediates cell adhesion. FEBS Lett. 2009; 583:2077-2082. [PubMed: 19467350] 
59. Wang Z, Schey KL. Aquaporin-0 interacts with the FERM domain of ezrin/radixin/moesin proteins in the ocular lens. Invest Ophthalmol Vis Sci. 2011; 52:5079-5087. [PubMed: 21642618]

60. Gonen T, Sliz P, Kistler J, Cheng Y, Walz T. Aquaporin-0 membrane junctions reveal the structure of a closed water pore. Nature. 2004; 429:193-197. [PubMed: 15141214]

61. Cho SJ, et al. Aquaporin 1 regulates GTP-induced rapid gating of water in secretory vesicles. Proc Natl Acad Sci U S A. 2002; 99:4720-4724. [PubMed: 11917120]

62. Brown D. The ins and outs of aquaporin-2 trafficking. Am J Physiol Renal Physiol. 2003; 284:F893-901. [PubMed: 12676734]

63. Nozaki K, Ishii D, Ishibashi K. Intracellular aquaporins: clues for intracellular water transport? Pflugers Arch. 2008; 456:701-707. [PubMed: 18034355]

64. Sugiya H, Fukushima M, Hashimoto S. Role of aquaporins and regulation of secretory vesicle volume in cell secretion. J Cell Mol Med. 2008; 12:1486-1494. [PubMed: 18194450]

65. Tung JJ, Hobert O, Berryman M, Kitajewski J. Chloride intracellular channel 4 is involved in endothelial proliferation and morphogenesis in vitro. Angiogenesis. 2009; 12:209-220. [PubMed: 19247789] 


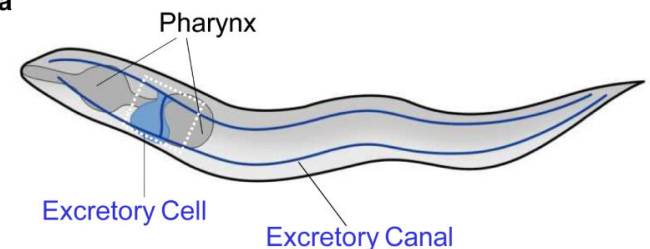

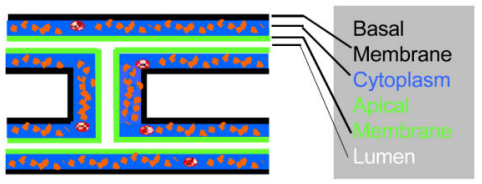

Excretory Canal

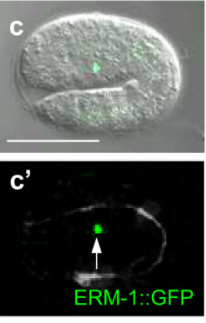

erm-1(tm677) (parent tm677)
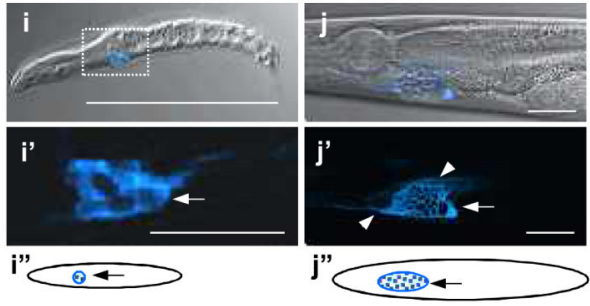

erm-1(tm677)/+
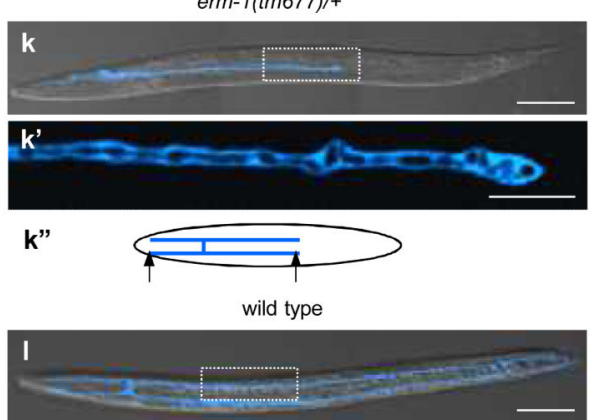

I'

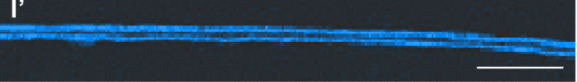

|" erm-1(tm677) (parent tm677/+)
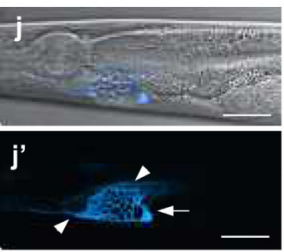

i"

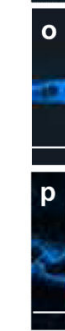

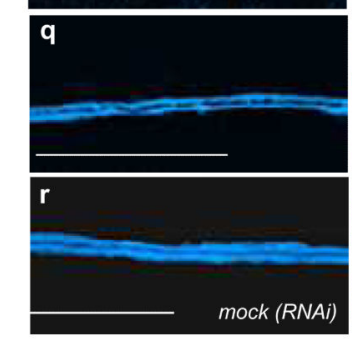

erm-1(RNAi)
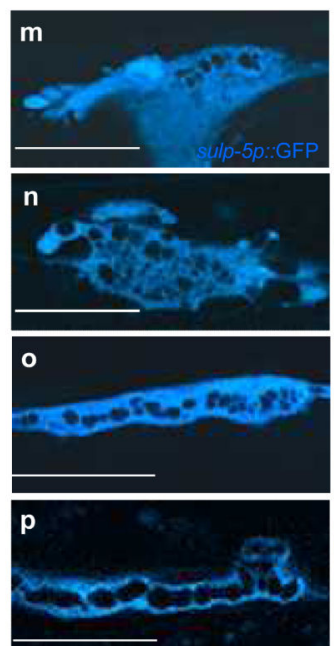

4

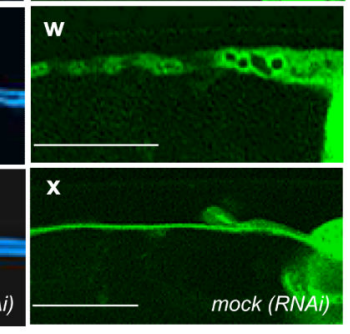

Figure 1. ERM-1 is required to expand the excretory-canal lumenal membrane and its actin undercoat

(a-b) Schematics of the C. elegans single excretory cell with canals (compare Fig. S1A-H). The C. elegans excretory system consists of five cells (excretory-, duct-, pore- and two gland cells) $)^{3}$, only the excretory cell is shown. The whole animal is shown in (a), and the outlined area is magnified in (b). Here and below, canal cytoplasm is displayed in blue, lumenal membrane/cytoskeleton in green, canalicular and endosomal vesicles in red (fluorophores are pseudo-colored accordingly, exceptions are indicated). 
(c-h') ERM-1 tracks lumenal membrane extension during canal morphogenesis (1.5-fold embryo to L1-larva, from c-h; compare Fig. 5a-d', S1I-J"). For clarity, intestinal

ERM-1::GFP is changed to gray in lower panel. Nascent canal lumen (arrows), direction of canal extension (bi-directional arrows) and canal tips (arrowheads) are indicated. Scale bars $25 \mu \mathrm{m}$, (for c-g in c' and h-h' in h).

(i-l') ERM-1 dose-dependently restricts canal extension. (i-i") Homozygous L1-larva (without maternal RNA): no canal extension and fluid in cell body (arrow). (j-j”)

Homozygous adult (maternal RNA present): no canal extension; note extended cell body (arrow) with lateral and anterior-posterior cusps (arrowheads) and cytoplasmic vacuoles (compare Fig. 1s for absence of actin lining). (k-k") Heterozygous adult: 1/2 posterior canal length; lumen is not contiguous ( $\mathrm{k}^{\prime}$, compare to $\left.\mathrm{m}-\mathrm{x}\right)$. (1-l') Wild-type adult: contiguous lumen (color-excluded stripe; l'). Scale bars $100 \mu \mathrm{m}$ (i-l), $15 \mu \mathrm{m}$ (i'-l').

$(\mathbf{m}-\mathbf{x})$ erm-l(RNAi) disrupts the formation of a contiguous lumen (m-r) and actin coat (s-x; compare Fig. S2D-O). Decreasing phenotypic severity from (m) through (q) and (s) through (w): (m) stretched cell body with few vacuoles, no canals; (n) accumulating unaligned vacuoles, no canals; (o) 1/4-extended canal with aligning vacuoles; (p) canal extended between $1 / 4$ and $1 / 2$-length with partially-fused vacuoles; note directional deviation where vacuoles are unaligned; (q) 1/2-extended canal with vacuolar outline of mostly contiguous lumen; (r) fully-extended canal and contiguous lumen; (s) cytoplasmic actin in cell body, vacuoles lack actin coat, no canal; (t) actin fragments (light grey in schematic inset) decorate single-standing vacuoles, partially-extended canal; (u) uniform actin-coating of unaligned but contacting vacuoles, partially-extended canal; (v) cytoplasmically-lined actin-coat (light grey in schematic inset) in aligning vacuoles; (w) increase of lumenal and decrease of cytoplasmic actin; ( $x$ ) full actin recruitment to contiguous lumenal membrane and loss of cytoplasmic actin. Representative confocal sections of cell bodies (m,n,s) and single arms of posterior canals (p-r, o includes part of anterior canal) and anterior canals (t-x; intestinal actin visible on right sides of images) of L4-stage-larvae or young-adults are shown. Scale bars $25 \mu \mathrm{m}$.

In all panels, anterior is left, posterior right, dorsal up, ventral down; boxed areas enlarged in (i', $\left.\mathrm{k}^{\prime}, \mathrm{l}^{\prime}\right)$ and schematics in (i", $\left.\mathrm{k}^{\prime}, \mathrm{l}^{\prime \prime}\right)$. sulp-5p::GFP shown in (i-r), pseudo-colored to blue. 


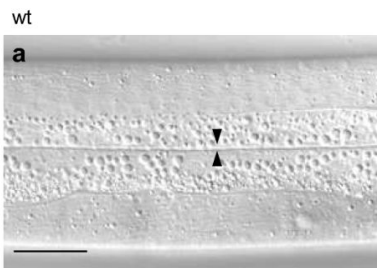

ERM-1[++]
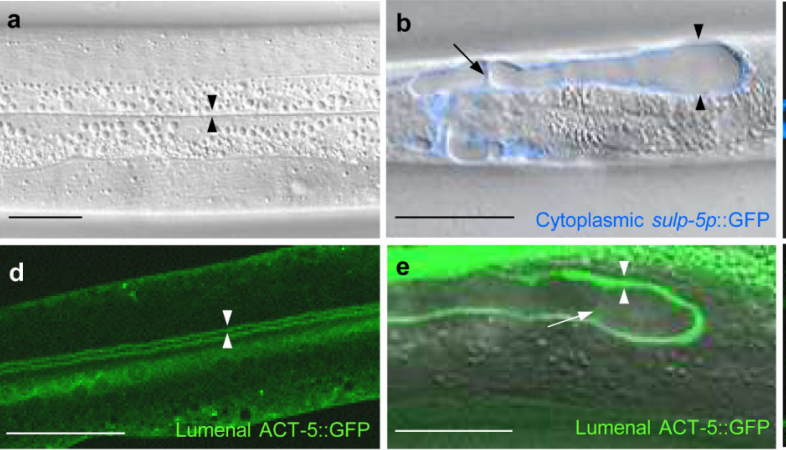

g

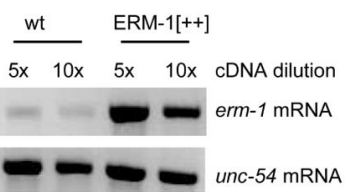

h

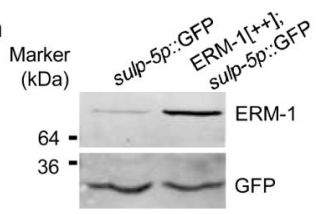

i

\begin{tabular}{|l|r|}
\hline Cytoskeletal and cytoskeleton related proteins & 624 \\
\hline Signaling and related proteins & 494 \\
\hline Receptors, ligands and related proteins & 254 \\
\hline Endo- and Exocytosis related proteins & 171 \\
\hline Channels and related proteins & 103 \\
\hline PDZ and FERM domain containing and related proteins & 63 \\
\hline Small GTPase and related proteins & 66 \\
\hline Mucin and related proteins & 71 \\
\hline Total & $\mathbf{1 8 4 6}$ \\
\hline
\end{tabular}

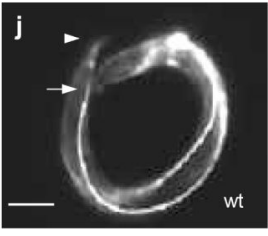

n

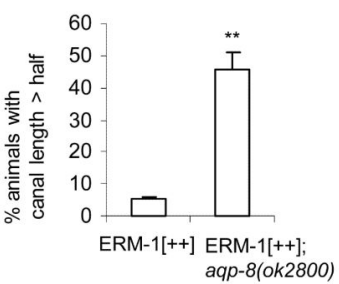

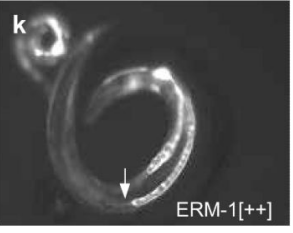
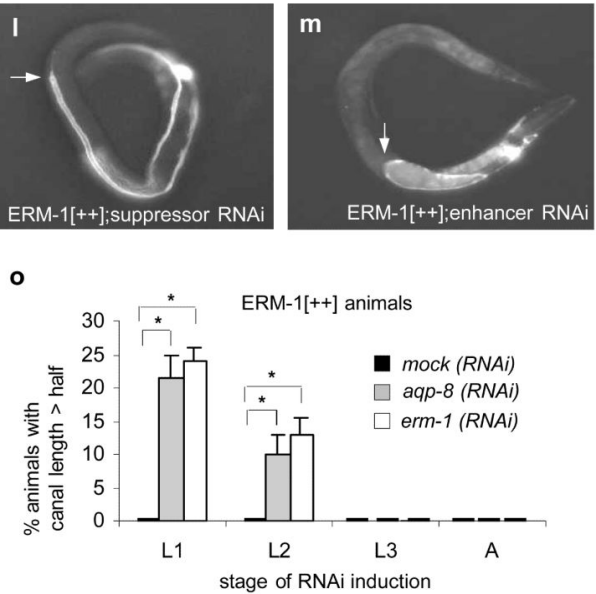

Figure 2. The ERM-1[++] cystic canal phenotype is suppressed by loss of AQP-8

Note that ERM-1 overexpression (shown here in Fig. 2) results in widened canals in adults, whereas ERM-1 depletion (shown in Fig. 1) results in "no-canals" or thin canals in L1larvae (Fig. 1, 2 images appear deceptively similar due to partial views of enlarged canal sections). (a-f) ERM-1[++] canal (compare Fig. S3A-F for different ERM-1[++] lines and dose-dependent increase in canal diameters). (a) Wild-type (wt) adult canal; lumen diameter is bracketed by arrowheads. (b) ERM-1[++] L3 cystic canal with wide lumen diameter (arrowheads) and crimped perimeter; an occasional occlusive septum is indicated by arrow. 
(c) ERM-1[++] lumen pockets framed by typical non-occlusive membrane septum (arrow) and leading cytoplasm without lumen (arrowhead). (d) Wild-type adult lumenal actin coat (single coat of posterior dorsal membrane sleeve bracketed by arrowheads). (e) ERM-1[++] thickened lumenal actin coat (arrowheads); canal septum is indicated by arrow (see Fig. S3K-O for ACT-5 quantification). (f) Excess actin beyond cyst tips (arrowheads; note that this is lumenal membrane undercoat, not cytoplasm (as shown in c). Scale bars $25 \mu \mathrm{m}$. Nomarski (a), Nomarski/confocal overlays (b,c) and confocal (c,d,f) images are shown. sulp-5p::GFP is pseudo-colored to blue.

(g) RT-PCR and (h) western blot, showing increased erm-1 mRNA and ERM-1 protein levels in ERM-1[++] (compare Fig. S3G,H).

(i) Broad categories of molecules targeted in the ERM-1[++] modifier screen. Protein classes were expanded from published in vivo and in vitro data of ezrin-radixin-moesininteracting molecules of all species. Corresponding C.elegans orthologs were targeted (Methods).

(j-m) ERM-1[++] modifier screen. Dissecting fluorescence microscopic images of vha-1p::gfp; rol-6p::rol-6(su1006) live animals are shown, as screened. Posterior canal tips are indicated by arrows, tail tip by arrowhead in wild-type (wt). Scale bar $100 \mu \mathrm{m}$.

(n) ERM-1[++] cystic and shortened canals are suppressed by a loss-of-function mutation in AQP-8 (compare Fig. S4A-B').

(o) Stage-specific suppression of the ERM-1[++] canal phenotype by post-embryonic aqp-8 and erm-1 RNAi, restricted to the L1-L2 early larval stage (full reversion to wild-type of ERM-1 [++] by standard erm- 1 RNAi is shown in Fig. S3I-J). A = adult.

Data in $(\mathrm{n}, \mathrm{o})$ presented as mean $\pm \mathrm{SD}\left(\mathrm{n}=3, * p<0.05,{ }^{*} p<0.01\right.$, two-tailed $t$-test $)$. 

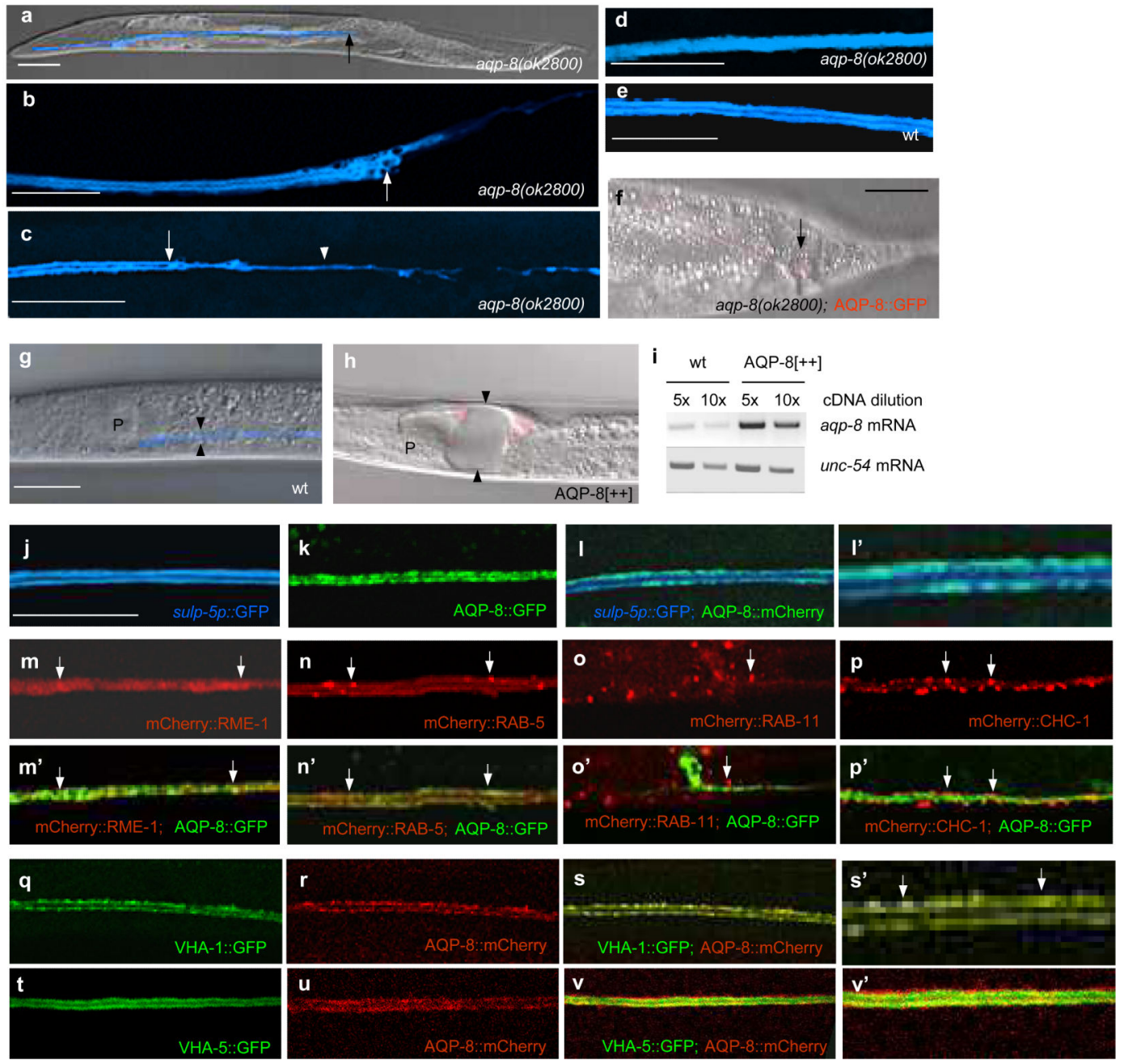

w

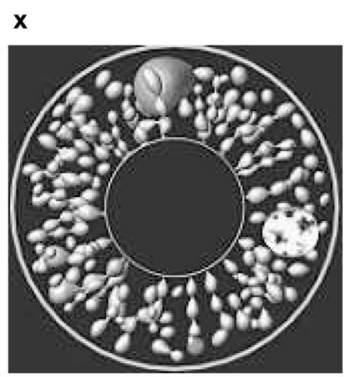

Figure 3. AQP-8 promotes excretory canal lumen expansion and localizes to canalicular vesicles (a-f) aqp-8(ok2800) canal phenotype. (a) Canal shortened to $1 / 2$ body length (arrow). (b) Vacuole accumulation at shortened canal tip (arrow). (c) Fully extended canal with premature lumen stop (arrow) and leading cytoplasm (arrowhead) with typical cytoplasmic islets. (d) Missing lumen in L4-larval canal (compare to wild-type, e). (f) Reversion to wildtype (canal extension to the tail, arrow) in aqp-8(ok2800); aqp-8p::aqp-8::gfp animal (ventral bent of canal is due to rol-6 background; GFP pseudo-colored to red; compare Fig. S5B). Scale bars $100 \mu \mathrm{m}$ in a, $25 \mu \mathrm{m}$ in b-f. 
(g,h) Higher copy numbers of a rescuing $a q p-8 p:: a q p-8:: g f p$ transgene (AQP-8[++], pseudo-colored to red, h) cause round cell-body-close cysts and widened canals (arrowheads at canal lumen boundaries; compare to wild-type [wt], g, and Fig. S5C-E'). P, pharynx.

Scale bar $25 \mu \mathrm{m}$.

Nomarski/confocal overlays (a,f-h), confocal projections (c,d,e) and confocal section (b) are shown. sulp-5p::GFP in (a-e,g), pseudo-colored to blue.

(i) RT-PCR showing increased aqp-8 mRNA levels in AQP-8[++] animals (compare Fig. $\mathrm{S} 5 \mathrm{~F})$.

(j-v') AQP-8 subcellular location in wild-type adult canals (only sulp-5p::GFP (j,1) and AQP-8::mCherry in (1) are pseudo-colored). Cytoplasmic canal GFP (j) is uniformly distributed throughout canal, whereas AQP-8 (k) labels dense but distinct and partially interconnected cytoplasmic puncta that are predominantly lumen distant (compare Fig. 5ii"'; double-labeled image shown in 1 is enlarged in l'). Endosomal vesicles (m-p') do not overlay AQP-8::GFP puncta (identical vesicles indicated by arrows in single, top, and merged images, bottom), are larger, sparse, ovoid and not connected (see model in w,x). In contrast, VHA-1::GFP (q-s) and VHA-5::GFP (t-v) mimic the AQP-8 expression pattern and partially overlap AQP-8::mCherry puncta (yellow in merged images in $\mathrm{s}$ and $\mathrm{v}$, enlarged in s' and v'). Confocal projections (j-l') and sections (m-v') of single canal arms are shown. Scale bar $25 \mu \mathrm{m}$, except enlarged views: (12 $\mu \mathrm{m}$ in l', $7.5 \mu \mathrm{m}$ in s' and $12 \mu \mathrm{m}$ in v'). $(\mathbf{w}-\mathbf{x})$ Angled (w) and end-on (x) views of a 3D excretory canal model with canalicular and endosomal vesicles shown (based on TEM and tomography, compare Fig. 7h-k, 8a-d). Small canalicular vesicles (white arrow) partially interconnect (arrowhead) and connect to the main lumen (black arrow) via tubular extensions. Endosomal vesicles are large, ovoid, isolated, and collect at the basal membrane (grey arrow). 
a

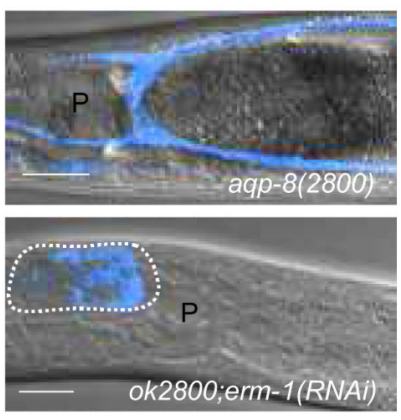

C

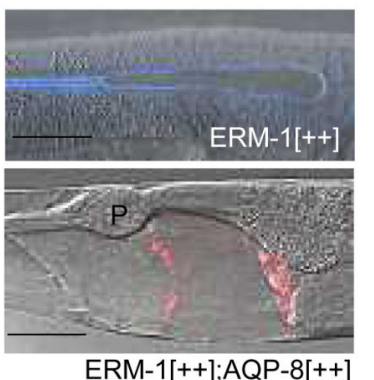

e

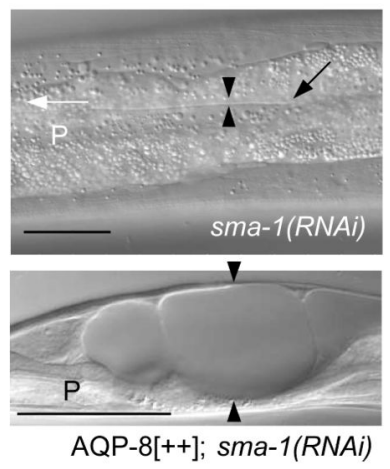

b
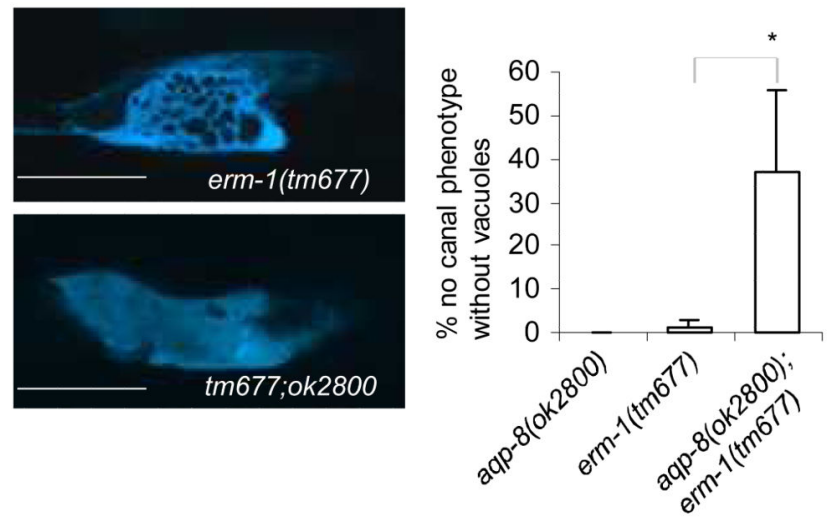

d
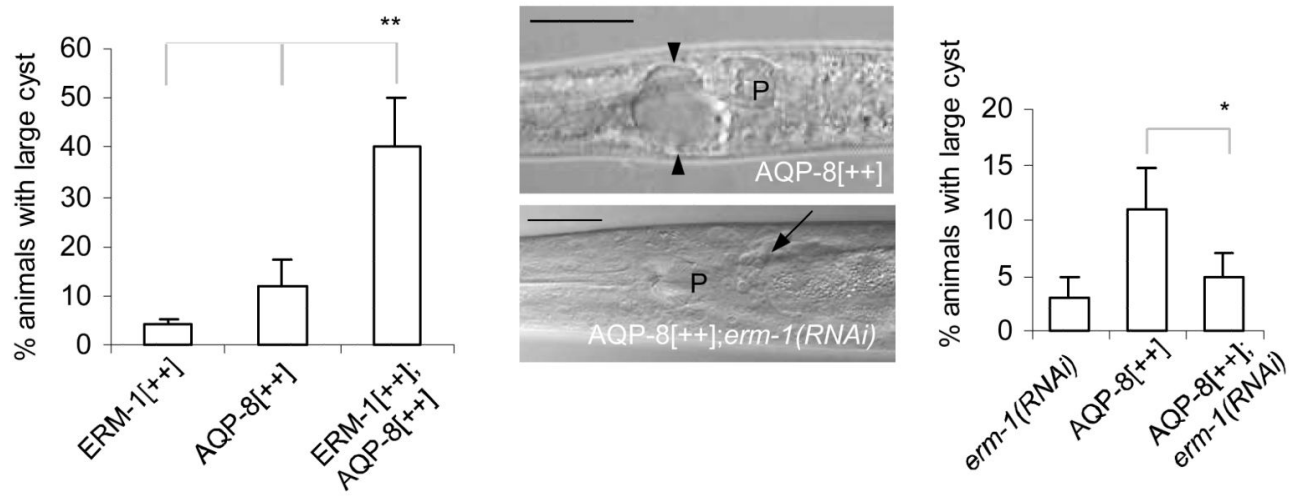

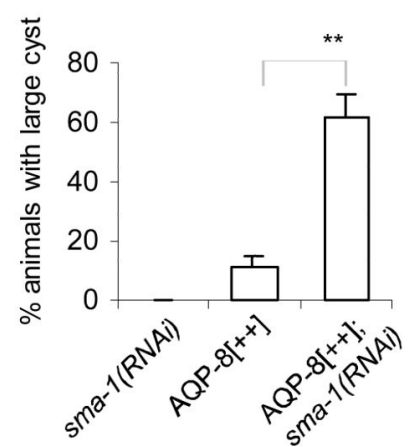

Figure 4. erm-1 and aqp-8 genetically interact in intracellular lumen morphogenesis (a) aqp-8(ok2800) enhances canal extension defects in erm-1(RNAi) animals. Left: aqp-8(ok2800) wild-type canal bifurcation at the cell body (top) and aqp-8(ok2800);erm-1(RNAi) 'no-canal' phenotype (cell body outlined by dotted line; bottom). Right: penetrance of 'no-canal' phenotype in single and double mutant/RNAi animals.

(b) aqp-8(ok2800) suppresses the formation of canal cell body vacuoles in erm-1(tm677). Left: vacuolated stretched erm-1(tm677) cell body (top), versus 
aqp-8(ok2800);erm-1(tm677) stretched cell body without vacuoles (bottom). Right: penetrance of canal cell bodies that have no vacuoles, in single and double mutants.

(c) ERM-1[++] enhances AQP-8 [++]-induced canal cysts. Left: ERM-1[++] cystic canal (top), and ERM-1[++];AQP-8[++] double transgenic cysts (bottom; cyst induced solely by AQP-8[++] shown in d). Right: penetrance of large cysts ( $>1 / 2$ body width, as shown in lower image on the left) in single and double transgenic animals.

(d) erm-1(RNAi) suppresses AQP-8[++]-induced cyst formation. Left: AQP-8[++] round cyst at the cell body (arrowheads, top), and AQP-8[++];erm-1(RNAi) cyst at the cell body (arrow, bottom). Right: penetrance of large cysts (>1/2 body width) in single and double transgenic/RNAi animals (erm-l(RNAi) cysts [first column] are derived from accumulating cytoplasmic vacuoles).

(e) smal-(RNAi) enhances AQP-8[++]-induced cysts. Left: sma-1(RNAi) moderately wide (arrowheads) and short canal ( $\sim 1 / 2$ body length; top, arrow at canal tip $)^{4}$ and AQP-8[+ +]; sma-1(RNAi) cyst (bottom, arrowheads; AQP-8[++]-induced cyst shown in d). Right: penetrance of large cysts ( $>1 / 2$ body width, as shown in lower image on the left) in single and double transgenic/RNAi animals.

Canals are labeled with vha-1p::GFP (pseudo-colored to blue), except ERM-[++];AQP-8[+ + ] animal in (c), labeled with AQP-8::GFP, pseudo-colored to red. P, pharynx. Nomarski images (d,e), Nomarski/confocal overlays (a,c) and confocal images (b) are shown. Scale bars $25 \mu \mathrm{m}$. Data presented as mean $\pm \mathrm{SD}\left(\mathrm{n}=5\right.$ in a,e; $\mathrm{n}=3$ in $\mathrm{b}-\mathrm{d},{ }^{*} p<0.05$, $* * p<0.001$, two-tailed $t$-test). 

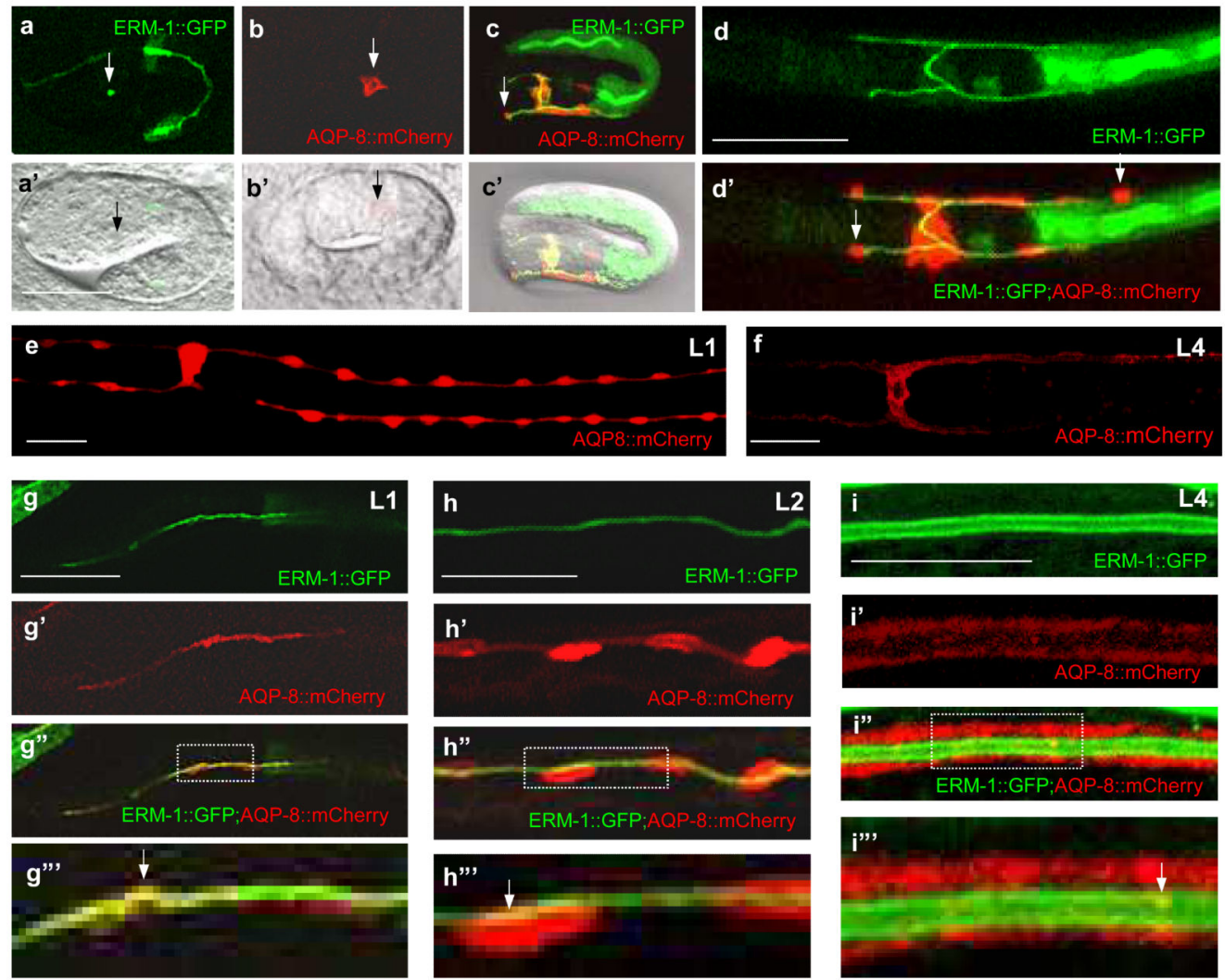

j aqp-8 cDNA fragments $\mathrm{X}$ erm-1Full-length cDNA

erm-1 cDNA fragments $X$ aqp-8 Full-length cDNA
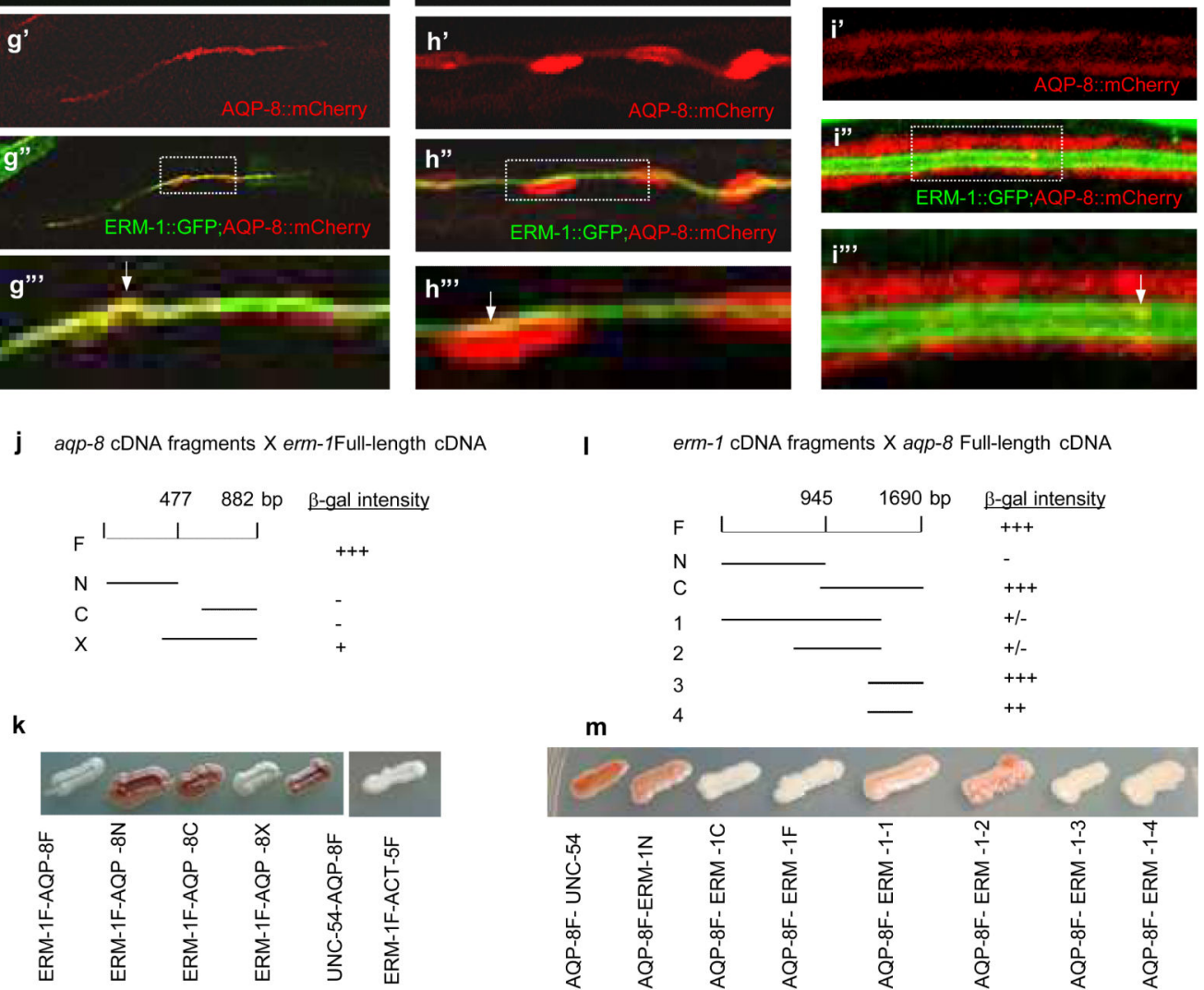

Figure 5. AQP-8 and ERM-1 transiently colocalize during excretory canal development and physically interact in yeast-two-hybrid assays

(a-d') Wild-type ERM-1 and AQP-8 expression during embryonic canal extension. (a,a') ERM-1::GFP appears coincident with the canal cell lumen in the 1.5-fold embryo (arrows). (b,b') AQP-8::mCherry appears later, in the canal cell cytoplasm of the 2.5-fold embryo (arrows). (c-d') Cytoplasmic AQP-8::mCherry moves to canal tips to the front of lumenal ERM-1::GFP in the 3-fold embryo (c,c'), and just-hatched L1 larva (d,d',arrows). (e,f) AQP-8 expression peaks in varicosities spaced at intervals along the canal during its active growth phase in L1-L2 larvae. Note bright AQP-8::mCherry expression in L1-larva 
(e) versus dim expression in L4-larva (f; image brightness is increased; actual AQP-8 levels in L4 and adult animals are undetectable with equivalent laser settings).

(g-i"') ERM-1 and AQP-8 expression during larval canal extension. (g-g"') ERM-1 and AQP-8 expression overlap at puncta (arrow) along L1 larval canal lumens. The outlined area in (g") is enlarged in (g"'). (h-h"') Lumenal ERM-1/AQP-8 overlap (arrow) is increased in, and largely restricted to, varicosities in L2 larval canals. The outlined area in (h") is enlarged in (h'"). (i-i") Sparse AQP-8/ERM-1 overlap in L4 (images in i" and I"' are overexposed to reveal the absence of overlap). The outlined area in (i") is enlarged in (i'"). Single yellow spot (arrow) is a rare colocalizing punctum.

Confocal images with corresponding Nomarski/confocal overlays (a'-c') are shown, single canal arm sections in (g-i"'). Scale bars $25 \mu \mathrm{m}$.

(j-m) ERM-1 physically interacts with AQP-8 and ACT-5 in yeast-two-hybrid assays. (j,k) Interactions of full-length ERM-1 with indicated AQP-8 fragments and ACT-5. $(1, \mathrm{~m})$ Interactions of full-length AQP-8 with indicated ERM-1 fragments. Negative control is derived from a $1 \mathrm{~kb}$ unc-54/myosin genomic DNA (exon 5). (k,m) White colony indicates physical interaction; purple colony indicates no interaction. See Fig. S8D,D' for betagalactosidase assays. $\mathrm{F}=$ full-length protein, $\mathrm{N}=\mathrm{N}$-terminal fragment, $\mathrm{C}=\mathrm{C}$-terminal fragment, $\mathrm{X}$ and $1-4=$ other fragments. 

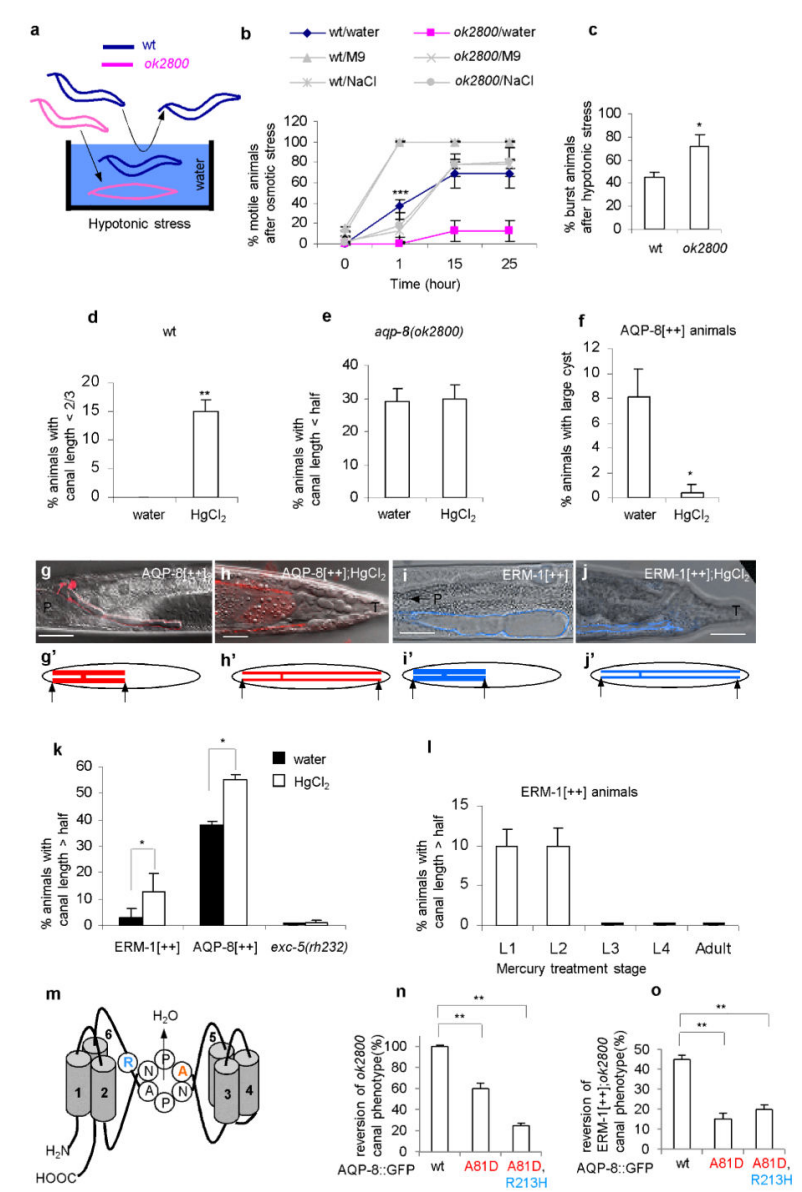

Figure 6. AQP-8 functions as a water-channel in canal morphogenesis

(a-c) aqp-8(ok2800) animals are sensitive to hypotonic stress. (a) Wild-type and aqp-8(ok2800) animals were soaked in water, 0.5 M salt solution or M9 (C. elegans growth medium; Methods) for 8 hours. (b) Percentage of animals recovering motility upon removal onto OP50 plates. (c) Percentage of animals bursting at the vulva secondary to fluid accumulation after incubation in water.

(d-l) Mercury affects canal extension and suppresses AQP-8[++] and ERM-1[++] canal cysts. (d,e) Penetrance of canal extension defects in wild-type (d) and aqp-8(ok2800) (e) after mercury treatment. (f) AQP-8[++] cysts (see Fig. 4d) are almost completely suppressed by mercury. (g-k) Suppression of AQP-8[++] and ERM-1[++] but not exc-5(rh232) canal extension defects by mercury. (k) Percentage of animals with canal re-extension. (1) Stagespecific suppression of the ERM-1[++] canal phenotype by post-embryonic mercury treatment.

Mercuric chloride was added to worm plates as described in Methods. Nomarski/confocal overlays and corresponding schematics shown in g-j', AQP-8::GFP pseudo-colored to red, sulp-5p::GFP in $(\mathrm{i}, \mathrm{j})$ pseudo-colored to blue. $\mathrm{P}=$ pharynx, $\mathrm{T}=$ tail. Data presented as mean $\pm \mathrm{SD}(\mathrm{n}=3, * p<0.05, * * \mathrm{p}<0.001$, two-tailed $t$-test $)$.

(m-o) Mutations in the channel region suppress the ability of AQP-8::GFP to revert aqp-8(ok2800) shortened canals to wild-type and ERM-1[++] aqp-8(ok2800) re-extended canals back to cystic canals. (m) AQP-8 channel structure with predicted transmembrane 
domains 1-6 (amino acids 23-45, 55-77, 97-119, 152-174, 181-203 and 234-256, created by SMART, http://smart.embl-heidelberg.de/) and signature NPA channel motif. Locations of mutated residues A81D and $\mathrm{R} 213 \mathrm{H}$ are indicated by orange and blue colors, respectively. (n-o) Percentage of animals with reversion of their respective phenotypes after transformation with wild-type versus mutated $a q p-8$ - $g f p$ transgenes. Data presented as mean $\pm \mathrm{SD}(\mathrm{n}=3, * * \mathrm{p}<0.001$, two-tailed $t$-test $)$. 

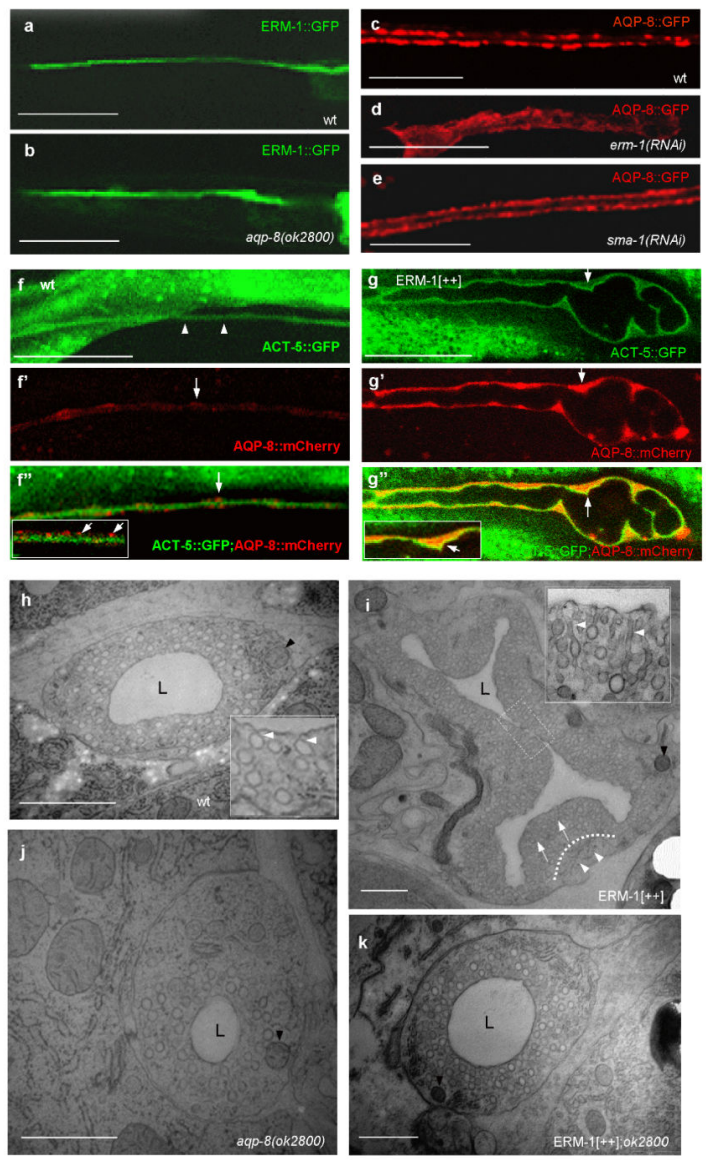

Figure 7. ERM-1 recruits AQP-8 to the lumen and increases the canalicular-lumenal membrane connection

(a-e) erm-1(RNAi) interferes with lumenal AQP-8 recruitment during larval canal extension. Lumenal ERM-1::GFP localization (wild-type [wt], a) is unchanged in aqp-8(ok2800) larvae (b). Canalicular AQP-8::GFP (pseudo-colored to red, c) disintegrates and recedes from the lumen in erm-1(RNAi) larvae (d), but not in sma-1(RNAi) larvae (e).

(f-g') ERM-1 overexpression recruits both AQP-8 and ACT-5 to the larval and adult canal lumen. Lumenal ACT-5::GFP (wild-type in $\mathrm{f}$, arrowheads) is thickened in ERM-1[++] canals (g, arrow; compare Fig. 2d-f). Low-level wild-type canalicular AQP-8::mCherry expression (f', arrow) is increased in ERM-1[++] canals and accumulates between cysts (g', arrow; compare Fig. S8G-H"). ACT-5::GFP and AQP-8::mCherry overlap at the lumen in ERM-1[++] (yellow in g", arrows) but not wild-type canals (f', arrows). Insets show higher magnification of lumenal area (inset in $\mathrm{f}$ " is from a different animal). Compare Tab. S2 for quantification of ACT-5/AQP-8 overlap.

Single canal arm confocal projections are shown in (a-e), sections in f-g", partially obscured by intestinal ACT-5::GFP staining in (f,f'). Scale bars $25 \mu \mathrm{m}$.

(h-k) HPF-TEM cross sections of wild-type (wt), fgIs2(erm-1p::erm-1) (ERM-1[++]), aqp-8(ok2800), and ERM-1[++]; aqp-8(ok2800)) excretory canal arms (compare Fig. 8a-d). (h) Wild-type canal section with dense, mostly single-standing canalicular vesicles. (i) ERM-1[++] canal section with deformed lumen, undulating lumenal membrane and cytoplasmic cushions with an increase in total number of lumen-connected vesicles. Shift of 
canalicular membrane system towards the lumen is indicated by arrows; dotted line separates canaliculi-rich lumenal-, from canaliculi-poor basal, area [arrowheads]). Inset: higher magnification image of ERM-1[++] canaliculi extending long tubules (arrowheads) towards the lumen that are not seen in wild-type (compare inset in h and Fig. 8a-d). (j) $a q p-8(o k 2800)$ canal with smaller lumen diameter and a reduced number of interconnected canalicular vesicles. (k) ERM-1[++] aqp-8(ok2800) canal exhibits features similar to wildtype.

Examples of isolated endosomes at typical basal locations are indicated by black arrowheads. L, lumen. Scale bars $1.0 \mu \mathrm{m}$. 

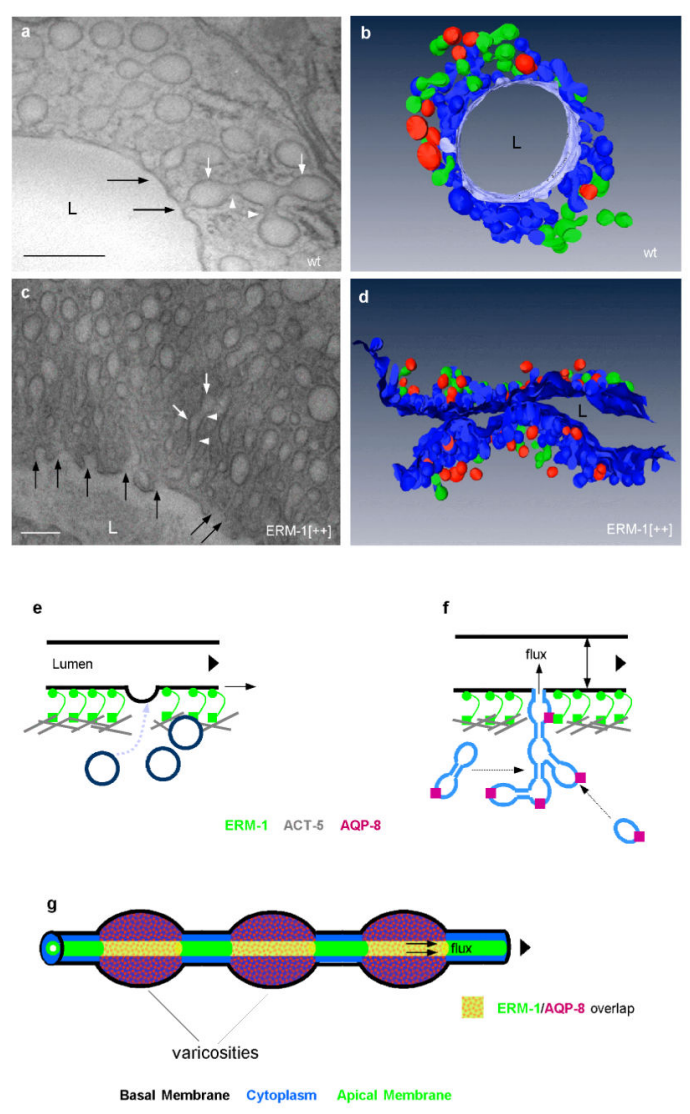

Figure 8. Tomographic analysis of the ERM-1[++] effect on the canalicular-lumenal interface and a model of the ERM-1/AQP-8 function in excretory canal lumen extension

(a,c) High-magnification images of HPF-TEM sections of the lumenal surface of wild-type (wt, a) and ERM[++] canals (c). Note increase in canaliculi-lumen connection (black arrows, also see Fig. S8I,J), length of canaliculi-lumen tubules, canalicular vesicle-vesicle connections (arrowheads) and density of canalicular vesicles (white arrows) in (c). L, lumen. Scale bars $0.25 \mu \mathrm{m}$.

(b,d) Tomographs showing canalicular vesicles connected to the lumen (blue), interconnected vesicles not connected to the lumen (green), and isolated vesicles, not connected to the lumen nor to each other (red). Note the increase in the number of lumenconnected canalicular vesicles in ERM-1[++] animals (d, corresponding to boxed area in Fig. 7i), as compared to wild-type (b, compare to Fig. 7h).

(e-g) Model. (e) High-magnification view. ERM-1 connects the lumenal membrane (black) to submembranous and perhaps vesicular actin (grey rods) via its respective globular $\mathrm{N}$ terminus (green circles) and C-terminus (green squares), thereby recruiting, incorporating, and coating lumenal-membrane-forming vesicles (bluegreen circles) with consequent membrane expansion (arrow) and lumen extension (arrowhead). (f) ERM-1 also connects the lumenal membrane (black) through its C-terminus (green squares) to the integral vesicular-membrane channel AQP-8 (red squares), thereby recruiting, but not integrating, canalicular vesicles (blue ovals) into the expanding lumenal membrane. This interaction (directly or indirectly) promotes translumenal flux with consequent diameter expansion 
(vertical arrow) and subsequent lumen extension (arrowhead). ACT-5 (shown in e) and AQP-8 (shown in $\mathrm{f}$ ) may compete for the $\mathrm{C}$ terminus of ERM-1. (g) Low magnification view. During the active phase of canal extension AQP-8 expression peaks in canal varicosities that successively arise along the extending canal, thereby shaping its overlap with ERM-1 into peri-lumenal cuffs. This temporal and spatial restriction of the ERM-1AQP-8 interaction could result in fluid pulses (from the cytoplasm into the developing lumen) that propel directional lumen and canal extension (arrowhead). 